(2) Open Access Full Text Article

ORIGINALRESEARCH

\title{
Impact of Hyperinsulinemia on Long-Term Clinical Outcomes of Percutaneous Coronary Intervention in Patients without Diabetes Who Have Acute Myocardial Syndrome
}

\section{Qiang Tan \\ Ming Chen \\ Jia Hao \\ Kun Wei}

Department of Cardiology, Qinhuangdao First Hospital, Hebei Medical University, Qinhuangdao, Hebei Province, People's Republic of China
Correspondence: Qiang Tan Department of Cardiology, Qinhuangdao First Hospital, Hebei Medical University, No. 258 of Wenhua Road, Qinhuangdao, Hebei Province, 066000, People's Republic of China

Email qhdtanqiang@aliyun.com
Background and Objectives: Hyperinsulinemia plays a key role in the development of cardiovascular impairment in patients with metabolic syndrome. The aim of this study was to evaluate the influence of hyperinsulinemia on long-term clinical outcomes of percutaneous coronary intervention (PCI) in patients without diabetes mellitus who have acute myocardial syndrome (ACS).

Methods: Between March 2016 and January 2019, we enrolled 468 patients with ACS and without diabetes mellitus who received primary PCI. Patients were divided into a low-insulin group $(\mathrm{n}=157)$, medium-insulin group $(\mathrm{n}=154)$, and high-insulin group $(\mathrm{n}=157)$ according to tertiles of fasting insulin level. The primary endpoint was major adverse cardiac events (MACE; all-cause death, non-fatal myocardial infarction, target vessel revascularization [TVR]) at 24 months. The secondary endpoint was angina hospitalization.

Results: Patients in the high-insulin group had an unfavorable prognosis, with a higher rate of MACE (34.39\%) than the low-insulin group (22.29\%) and medium-insulin group (23.37\%) at 24 months $(\mathrm{P}<0.05)$. This difference was mainly driven by the increase in TVR. The high-insulin group also had a higher rate of angina hospitalization than the low-insulin group. Cox proportional hazards regression showed that high-insulin level (hazard ratio [HR] 1.870, 95\% confidence interval [CI] 1.202-2.909), small-vessel lesion (HR 1.713, 95\% CI 1.111-2.642), bifurcation lesion (HR 3.394, 95\% CI 2.033-5.067), SYNTAX score (HR 1.084, 95\% CI $1.039-1.130$ ), and stent length (HR 1.017, 95\% CI 1.002-1.032) increased the incidence of MACE in patients with ACS and without diabetes who underwent PCI.

Conclusion: Hyperinsulinemia might be a valid predictor of clinical outcomes in patients with ACS undergoing PCI.

Keywords: percutaneous coronary intervention, hyperinsulinemia, acute myocardial syndrome, major adverse cardiac events

\section{Introduction}

Metabolic syndrome (MetS) has a high prevalence worldwide. ${ }^{1}$ MetS is associated with the development of coronary atherosclerosis, plaque instability, and cardiovascular events in patients with and without type 2 diabetes. ${ }^{2,3}$ The core components of MetS are insulin resistance (IR) and hyperinsulinemia. ${ }^{4}$ A new concept of selective IR proposes that tissues become resistant to the effect of insulin on glucose transport but remain sensitive to its lipogenic effect. ${ }^{5}$ IR may be 
compensatory in the body's response to preventing MetS. Hyperinsulinemia is more common than IR and may play a primary role in the development of cardiovascular impairment in patients with MetS. ${ }^{6}$

Previous studies show that hyperinsulinemia is associated with hypertension, obesity, and dyslipoproteinemia, which are risk factors for coronary artery disease. ${ }^{7,8}$ Other studies have indicated that hyperinsulinemia interferes with arteriolar vasodilation and bioavailability of nitric oxide (NO) in obese, insulin-resistant, and healthy individuals. ${ }^{4,9}$ Our previous study demonstrated that hyperinsulinemia impairs the functions of endothelial progenitor cells, which play a key role in maintaining endothelial function and vascular repair. ${ }^{10}$ However, the impact of hyperinsulinemia on major clinical outcomes following percutaneous coronary intervention (PCI) is largely unknown.

The aim of this study was to evaluate the impact of hyperinsulinemia on major clinical outcomes in patients with acute myocardial syndrome (ACS) undergoing PCI. Many studies have shown that diabetes mellitus is associated with ischemic events in patients undergoing PCI. ${ }^{11-13}$ Therefore, to avoid the influence of hyperglycemia on clinical outcomes of PCI, we excluded patients with diabetes mellitus in this study.

\section{Methods}

\section{Study Population}

This was a retrospective observational study. Between March 2016 and January 2019, patients with ACS who received PCI were retrospectively enrolled. The inclusion criteria were 1) patients at least 18 years old who had ACS; ACS was defined as ST segment elevated myocardial infarction (STEMI), non-ST segment elevated myocardial infarction (MI), and unstable angina. ${ }^{14}$ 2) Patients who underwent primary PCI with a drug-eluting stent (DES). The following were excluded from the study: 1) patients diagnosed with diabetes mellitus, defined according to American Diabetes Association criteria. ${ }^{15}$ 2) Patients without previously known diabetes but with glycated hemoglobin A1c $>6.5 \%$ on admission. 3) Patient angiography showing in-stent restenosis. 4) Patients who used insulin or insulin sensitizer within the past 3 months.

This study was approved by the ethics committee of Qinhuangdao First Hospital, and all patients provided their written informed consent.

\section{Biochemical Examination}

Venous blood samples were collected after an overnight fast. Plasma glucose and insulin were measured as well as other parameters including cholesterol, triglyceride (TG), low-density lipoprotein cholesterol (LDL-C), high-density lipoprotein (HDL-C), creatinine, high-sensitivity C-reactive protein, troponin-I, and B-type natriuretic peptide. Fasting insulin (FINS) levels were measured using a double-antibody sandwich immunoassay (Elecsys 1010 analyzer, Roche Diagnostics, Mannheim, Germany). IR was assessed using the homeostasis model assessment for insulin resistance (HOMA-IR) and the following formula: HOMA-IR $(\mathrm{mmol} / \mathrm{L} \times \mu \mathrm{U} / \mathrm{mL})=$ fasting glucose $(\mathrm{mmol} / \mathrm{L}) \times$ fasting insulin $(\mu \mathrm{U} / \mathrm{mL}) / 22.5 .^{16}$

\section{Interventional Procedure}

Patients were administered $300 \mathrm{mg}$ of aspirin and a loading dose of $300 \mathrm{mg}$ clopidogrel or $180 \mathrm{mg}$ ticagrelor. Coronary angiography and percutaneous coronary intervention were performed in all patients via a transradial or transfemoral approach. The angiographic findings were analyzed using a quantitative coronary angiography (QCA) system (GEQCA, CentricityAI1000-GEMnetVersion4.1.15.07). With the outer diameter of the contrast-filled catheter as the calibration standard, the minimal lumen diameter and lesion length were measured on diastolic frames. The SYNTAX score was calculated for each patient according to the results of coronary angiography.

\section{Follow-Up and Study Endpoints}

All patients received aspirin (100mg QD) during followup and clopidogrel (75mg QD) or ticagrelor (90mg BID) for at least one year. Patients underwent clinical observation for at least 24 months. Clinical follow-up was performed at 1, 6, 12, and 24 months.

The primary endpoint was major adverse cardiac events (MACE), including overall death, non-fatal MI, and target vessel revascularization (TVR). MI was diagnosed with an elevation in serum creatine kinase or troponin I three times the upper limit of normal, together with chest pain lasting more than 30 minutes. ${ }^{17}$ TVR was defined as any repeat revascularization of the stenttreated vessel. ${ }^{17}$ The secondary endpoint was angina pectoris requiring hospitalization. 


\section{Statistics Analysis}

All statistical analyses were performed using SPSS17 (SPSS, Chicago, IL, USA). Continuous variables are presented as mean \pm standard deviation if consistent with a normal distribution; otherwise, these are presented as median and interquartile range. Categorical variables are expressed as numbers and percentages. One-way analysis of variance or the Kruskal-Wallis $H$-test was applied to analyze differences in continuous variables between groups. Categorical variables were compared with the $\chi^{2}$ or Fisher's exact test.

Cox proportional hazards regression analysis was conducted to estimate the hazard ratios (HR) and their 95\% confidence intervals (CI) of developing the primary endpoint. Variables with a $\mathrm{P}$ value $<0.10$ in the univariate analysis were included in a multivariate analysis. MACE incidence rates of 24 months were estimated using the Kaplan-Meier method. A probability value $<0.05$ was considered statistically significant.

\section{Results}

The enrolled patients were divided into three groups according to tertiles of FINS level (T1, low-insulin group: $\mathrm{FINS}<7.89 \mu \mathrm{IU} / \mathrm{mL}$; T2, medium-insulin group: $7.89 \mu \mathrm{IU} / \mathrm{mL} \leq \mathrm{FINS}<14.33 \mu \mathrm{IU} / \mathrm{mL} ; \quad \mathrm{T} 3, \quad$ high-insulin group: FINS $\geq 14.33 \mu \mathrm{IU} / \mathrm{mL}$ ). Patients' baseline clinical characteristics are shown in Table 1. The three groups were balanced in terms of age, sex, family history, hypertension, smoking, prior MI, and clinical presentation. There were no significant differences in laboratory characteristics such as left ventricular ejection fraction (LVEF), cholesterol, TG, and LDL-C. Patients in the lowinsulin group had higher HDL-C than those in the highinsulin group $(P<0.05)$. BMI, waistline, HOMA-IR, and serum urine in the high-insulin group were higher than those in the low-and medium-insulin groups. The angiographic and procedural characteristics are shown in Table 1. The target vessel showed no significant difference among the three groups. QCA analysis revealed that the high-insulin group had longer lesion length and longer stents than the low-insulin group. SYNTAX scores were higher in the high-insulin group than in the low-insulin group. Patients in the high-insulin group also had more target vessel stenosis and bifurcation lesions than those in the low-insulin group.

The baseline clinical characteristics of patients, stratified by the primary endpoint, are summarized in Table 2 .
Compared with the event-free group, patients with MACE had higher levels of FINS. Patients with MACE also showed higher rates of prior myocardial infarction and higher values for the HOMA-IR, BMI, creatinine, and waistline measures. As shown in Table 2, patients with MACE had smaller vessel diameter, higher SYNTAX scores, and longer lesion length.

Clinical follow-up was completed in all surviving patients. Kaplan-Meier survival analyses showed that patients in the high-insulin group had an unfavorable prognosis, with higher rates of MACE and angina hospitalization than patients in the other two groups at 24 months (Figures 1 and 2). As shown in Table 3, the incidence of the primary endpoint in the high-insulin group was significantly higher than that in the low-insulin group $(\mathrm{P}<0.05)$. This difference was mainly driven by the increase in TVR. However, the incidences of overall death and non-fatal MI during follow-up were similar among the FINS tertiles.

Multivariate analysis and predictors for MACE within 24 months after PCI are presented in Table 4 and Supplementary Table 1 . As a categorical variable, hyperinsulinemia (T3, FINS $\geq 14.33 \mu \mathrm{IU} / \mathrm{mL}$ ) showed an increased HR for the incidence of MACE (HR2.586, 95\% CI 1.694-3.950; $\mathrm{P}=0.000$ ) in univariate analysis. After adjustment for multiple confounders, multivariate analysis showed a similar result (HR1.870, 95\% CI 1.202-2.909; $\mathrm{P}=0.001)$. Multivariate Cox regression also revealed that bifurcation lesion, small-vessel lesion, stent length, and SYNTAX score were associated with an increased HR for MACE in patients with ACS after PCI (all $\mathrm{P}<0.05$ ).

Insulin level as a continuous variable was associated with an HR of 1.062 (95\% CI1.025-1.010; $\mathrm{P}=0.001)$ in univariate analysis. However, in multivariate analysis, the association between insulin level and MACE was not significant after adjusting for confounding factors (HR1.036, 95\% CI 0.997-1.077, $\mathrm{P}=0.071)$ (Supplementary Table 2).

\section{Discussion}

The main finding of this study is that a high insulin level was associated with an increased risk of MACE in patients with ACS and without diabetes mellitus who were undergoing PCI. Hyperinsulinemia might be a valid predictor of clinical outcomes in patients with ACS undergoing PCI.

Weakened insulin signaling or insulin resistance, together with the associated diminution in glucose 
Table I Comparison of Clinical and Angiographic Characteristics According to FINS Status

\begin{tabular}{|c|c|c|c|c|c|}
\hline & Low Insulin $(n=157)$ & Medium Insulin $(n=\mid 54)$ & High Insulin $(n=157)$ & $\mathbf{F}$ or $\chi^{2}$ & $P$ value \\
\hline Age & $62.59 \pm 8.83$ & $62.01 \pm 9.15$ & $59.21 \pm 10.51$ & 0.255 & 0.775 \\
\hline Gender (M/F) & $104 / 53$ & $104 / 50$ & $118 / 39$ & 3.444 & 0.179 \\
\hline Current smoker & 77 (49.04\%) & 77 (50\%) & $76(48.41 \%)$ & 0.08 & 0.961 \\
\hline Family history & $58(36.94 \%)$ & $54(35.06 \%)$ & $54(34.39 \%)$ & 0.239 & 0.887 \\
\hline Hypertension & $102(64.86 \%)$ & $95(61.68 \%)$ & I0I (64.33\%) & 0.406 & 0.816 \\
\hline Prior MI & 18 (1 I.46\%) & II (7.14\%) & $14(8.91 \%)$ & 1.761 & 0.415 \\
\hline Clinical presentation & & & & 4.337 & 0.362 \\
\hline STEMI & 17 & 14 & 24 & & \\
\hline Non-STEMI & 25 & 30 & 31 & & \\
\hline Unstable angina & 115 & 110 & 102 & & \\
\hline Grace score & $74.44 \pm 21.36$ & $75.05 \pm 19.23$ & $75.21 \pm 19.01$ & 0.047 & 0.954 \\
\hline $\mathrm{TC}(\mathrm{mmol} / \mathrm{L})$ & $4.45 \pm 1.03$ & $4.46 \pm 2.22$ & $4.26 \pm 1.05$ & 0.785 & 0.457 \\
\hline $\mathrm{TG}(\mathrm{mmol} / \mathrm{L})$ & $1.72 \pm 1.20$ & $1.89 \pm 1.98$ & $2.16 \pm 1.22 *$ & 2.848 & 0.059 \\
\hline LDL-C (mmol/L) & $2.48 \pm 0.8 I$ & $2.46 \pm 0.91$ & $2.34 \pm 0.81$ & 1.161 & 0.314 \\
\hline HDL-C (mmol/L) & $1.12 \pm 0.24$ & $1.06 \pm 0.23$ & $1.04 \pm 0.22 *$ & 4.514 & 0.012 \\
\hline Glucose $(\mathrm{mmol} / \mathrm{L})$ & $5.46 \pm 0.78$ & $5.42 \pm 0.76$ & $5.51 \pm 0.90$ & 0.405 & 0.667 \\
\hline $\mathrm{HbAlc}(\%)$ & $5.82 \pm 0.75$ & $5.86 \pm 0.48$ & $5.87 \pm 0.41$ & 0.096 & 0.909 \\
\hline Insulin (ulU/mL) & $6.14 \pm 1.41$ & $10.68 \pm 1.71 * *$ & $17.69 \pm 3.77 * * \#$ & 835.16 & 0 \\
\hline HOMA-IR & $1.31 \pm 0.60$ & $2.14 \pm 1.06 * *$ & $3.62 \pm 1.93 * * \#$ & 121.732 & 0 \\
\hline Creatinine $(\mu \mathrm{mol} / \mathrm{L})$ & $67.89 \pm 14.59$ & $67.24 \pm 15.55$ & $75.13 \pm 68.69$ & $\mathrm{I} .47 \mathrm{I}$ & 0.231 \\
\hline Troponin I (ng/mL) M (IQR) & $0.34(0.03-20.84)$ & $0.35(0.036-22.42)$ & $0.33(0.05-16.45)$ & 1.198 & 0.549 \\
\hline BNP (ng/mL) M (IQR) & $54.1(22.32-145.09)$ & 80.17 (31.5-234) & $56.0(21.07-255.4)$ & 3.193 & 0.203 \\
\hline $\mathrm{HCY}(\mathrm{mmol} / \mathrm{L})$ & $15.84 \pm 8.93$ & $17.64 \pm 9.27$ & $16.14 \pm 8.82$ & 1.188 & 0.306 \\
\hline Urine (mmol/L) & $332.04 \pm 84.97$ & $336.78 \pm 94.19$ & $370.56 \pm 101.70 * \#$ & 6.511 & 0.002 \\
\hline BMI & $24.31 \pm 2.82$ & $26.25 \pm 2.72 * *$ & $27.84 \pm 3.41 * * \#$ & 53.53 & 0 \\
\hline Waistline $(\mathrm{cm})$ & $88.61 \pm 11.33$ & $92.66 \pm 8.12^{*}$ & $94.33 \pm 8.52^{* *}$ & 5.811 & 0.003 \\
\hline LVD (mm) & $48.93 \pm 4.62$ & $49.15 \pm 4.36$ & $49.72 \pm 4.76$ & 0.971 & 0.38 \\
\hline LAD (mm) & $37.77 \pm 5.49$ & $38.25 \pm 4.51$ & $39.21 \pm 7.38$ & 1.898 & 0.151 \\
\hline LVEF (\%) & $65.53 \pm 7.32$ & $65.11 \pm 7.13$ & $64.19 \pm 8.88$ & 0.979 & 0.388 \\
\hline \multicolumn{6}{|l|}{ Target artery } \\
\hline Left main artery & 8 (5.09\%) & $6(3.89 \%)$ & 7 (4.45\%) & 0.261 & 0.877 \\
\hline Left anterior descending & $95(60.51 \%)$ & $84(54.54 \%)$ & $90(57.32 \%)$ & 1.134 & 0.567 \\
\hline Left circumflex artery & 22 (14.01\%) & 25 (16.23\%) & $23(14.64 \%)$ & 0.319 & 0.853 \\
\hline Right coronary artery & 31 (19.76\%) & $31(20.12 \%)$ & 37 (23.56\%) & 2.626 & 0.269 \\
\hline Bifurcation & 29 (18.47\%) & $33(21.42 \%)$ & $45(28.66 \%)^{*}$ & 4.891 & 0.087 \\
\hline Multivessel disease & II 7 (74.52\%) & 119 (77.27\%) & 124 (78.98\%) & 0.895 & 0.639 \\
\hline \multicolumn{6}{|l|}{ Target lesion } \\
\hline Reference diameter $(\mathrm{mm})$ & $2.91 \pm 0.43$ & $2.93 \pm 0.49$ & $3.01 \pm 0.58$ & 2.55 & 0.079 \\
\hline Lesion length $(\mathrm{mm})$ & $19.60 \pm 8.54$ & $20.36 \pm 8.28$ & $22.52 \pm 9.39 *$ & 4.69 & 0.01 \\
\hline Diameter stenosis (\%) & $89.19 \pm 10.76$ & $90.70 \pm 7.72$ & $91.75 \pm 0.63 *$ & 3.24 & 0.04 \\
\hline Syntax Score & $|0.8| \pm 4.2 \mid$ & $10.73 \pm 3.97$ & $12.79 \pm 5.03 * \#$ & 10.727 & 0 \\
\hline \multicolumn{6}{|l|}{ Characteristics of DES } \\
\hline Diameter $(\mathrm{mm})$ & $2.91 \pm 0.41$ & $2.98 \pm 0.42$ & $3.0 I \pm 0.4 I$ & 2.121 & 0.146 \\
\hline Length (mm) & $24.26 \pm|4.7|$ & $25.71 \pm 13.09$ & $28.87 \pm 12.24 *$ & 4.561 & 0.011 \\
\hline
\end{tabular}

Notes: $* P<0.05$ compared with low insulin group; ${ }^{* *} P<0.01$ compared with low insulin group; ${ }^{\#} P<0.05$ compared with medium insulin group; ${ }^{\#} P<0.0$ I compared with medium insulin group.

Abbreviations: FINS, fasting insulin; LVEF, left ventricular ejection fraction; LVD, left ventricular diameter; LA, left atrial diameter; STEMI, ST-segment elevation myocardial infarction; NSTEMI, non-ST segment elevation myocardial infarction; TC, cholesterol; TG, Triglyceride; LDL-C, low density lipoprotein-cholesterol; HDL-C, high-density lipoprotein cholesterol; BNP, B-type natriuretic peptide.; BMI, body mass index; HCY, Homocysteine; HOMA-IR, homeostasis model assessment for insulin resistance; DES, drug-eluting stent. 
Table 2 Clinical and Angiographic Characteristics of MACE and Non-MACE Group

\begin{tabular}{|c|c|c|c|c|}
\hline & MACE Group $(n=86)$ & Non-MACE $(n=382)$ & $\mathbf{F}$ or $\chi^{2}$ & $P$ value \\
\hline Age & $62.04 \pm 9.44$ & $61.14 \pm 9.64$ & 0.733 & 0.464 \\
\hline Gender (M/F) & $24 / 62$ & $62 / 320$ & 0.017 & 0.896 \\
\hline Current smoker & 46 (53.48\%) & $184(48.16 \%)$ & 2.830 & 0.093 \\
\hline Family history & 25 (29.06\%) & $|4|$ (36.9|\%) & 0.239 & 0.887 \\
\hline Hypertension & 54 (62.79\%) & 250 (65.44\%) & 0.278 & 0.598 \\
\hline Prior Ml & $19(22.09)$ & $24(6.28 \%)$ & 21.03 & 0.000 \\
\hline Clinical presentation & & & 0.780 & 0.677 \\
\hline STEMI & 11 & 44 & & \\
\hline Non-STEMI & 13 & 73 & & \\
\hline Unstable angina & 62 & 265 & & \\
\hline Grace score & $74.11 \pm 14.78$ & $76.84 \pm 14.24$ & 0.047 & 0.954 \\
\hline TC $(\mathrm{mmol} / \mathrm{L})$ & $4.18 \pm 0.95$ & $4.43 \pm 1.6 \mathrm{I}$ & 1.230 & 0.219 \\
\hline TG (mmol/L) & $1.90 \pm 1.02$ & $1.93 \pm 1.59$ & 0.139 & 0.890 \\
\hline LDL-C (mmol/L) & $2.37 \pm 0.73$ & $2.43 \pm 0.86$ & 0.616 & 0.539 \\
\hline HDL-C (mmol/L) & $1.05 \pm 0.18$ & $1.08 \pm 0.24$ & 1.146 & 0.253 \\
\hline Glucose $(\mathrm{mmol} / \mathrm{L})$ & $5.52 \pm 0.66$ & $5.45 \pm 0.84$ & 0.635 & 0.526 \\
\hline HbAlc (\%) & $5.77 \pm 0.52$ & $5.86 \pm 0.58$ & 0.096 & 0.909 \\
\hline Insulin (ulU/mL) & $13.2 \mid \pm 4.15$ & $11.09 \pm 5.10$ & 3.305 & 0.001 \\
\hline HOMA-IR & $2.6 I \pm 0.95$ & $2.30 \pm 1.05$ & 2.458 & 0.014 \\
\hline Creatinine $(\mu \mathrm{mol} / \mathrm{L})$ & $81.59 \pm 11.59$ & $67.57 \pm 15.24$ & 2.607 & 0.009 \\
\hline Troponin I (ng/mL) M (IQR) & $0.42(0.06-33.30)$ & $0.34(0.03-30)$ & 1.346 & 0.178 \\
\hline BNP (ng/mL) M (IQR) & 78.01 (22.10-258.14) & $46.39(22.77-145.09)$ & 1.405 & 0.160 \\
\hline $\mathrm{HCY}(\mathrm{mmol} / \mathrm{L})$ & $18.11 \pm 11.33$ & $\mid 6.15 \pm 8.41$ & 1.476 & 0.141 \\
\hline Urine $(\mathrm{mmol} / \mathrm{L})$ & $344.81 \pm 106.27$ & $346.43 \pm 92.47$ & 0.129 & 0.898 \\
\hline BMI & $26.42 \pm 4.15$ & $25.98 \pm 3.25$ & 2.520 & 0.012 \\
\hline Waistline $(\mathrm{cm})$ & $85.10 \pm 10.69$ & $79.01 \pm 13.36$ & 5.811 & 0.003 \\
\hline LVD (mm) & $49.74 \pm 4.91$ & $49.16 \pm 4.51$ & 0.932 & 0.352 \\
\hline LAD (mm) & $37.4 I \pm 3.84$ & $38.58 \pm 6.20$ & 1.438 & 0.151 \\
\hline LVEF (\%) & $63.59 \pm 9.28$ & $65.25 \pm 7.43$ & 0.979 & 0.388 \\
\hline \multicolumn{5}{|l|}{ Target artery } \\
\hline Left main artery & $7(8.13 \%)$ & $14(3.66 \%)$ & 3.279 & 0.070 \\
\hline Left anterior descending & 54 (62.79\%) & $215(56.28 \%)$ & 1.216 & 0.270 \\
\hline Left circumflex artery & II (II.62\%) & 59 (I5.44\%) & 0.389 & 0.533 \\
\hline Right coronary artery & 14 (16.27\%) & $93(24.34 \%)$ & 2.590 & 0.108 \\
\hline Bifurcation & 35 (40.69\%) & 72 (I8.84\%) & 19.002 & 0.000 \\
\hline Multivessel disease & 68 (79.06\%) & $294(76.96 \%)$ & 0.178 & 0.673 \\
\hline \multicolumn{5}{|l|}{ Target lesion } \\
\hline Reference diameter $(\mathrm{mm})$ & $2.82 \pm 0.41$ & $2.94 \pm 0.45$ & 2.608 & 0.009 \\
\hline Lesion length (mm) & $24.31 \pm 9.06$ & $20.06 \pm 8.60$ & 4.070 & 0.000 \\
\hline Diameter stenosis (\%) & $92.68 \pm 6.07$ & $90.07 \pm 9.43$ & 2.457 & 0.014 \\
\hline Small vessel $($ diameter $<2.75 \mathrm{~mm})$ & 47 (54.65\%) & $59(15.44 \%)$ & 56.591 & 0.000 \\
\hline Syntax Score & $|4| I \pm 4.78$. & $10.84 \pm 4.24$ & 6.292 & 0.000 \\
\hline \multicolumn{5}{|l|}{ The characteristics of DES } \\
\hline Diameter $(\mathrm{mm})$ & $2.93 \pm 0.39$ & $2.97 \pm 0.42$ & 2.455 & 0.015 \\
\hline Length $(\mathrm{mm})$ & $31.15 \pm 12.33$ & $25.24 \pm 13.46$ & 3.666 & 0.000 \\
\hline
\end{tabular}

Abbreviations: MACE, major adverse cardiac events; LVEF, left ventricular ejection fraction; LVD, left ventricular diameter; LA, left atrial diameter; STEMI, ST-segment elevation myocardial infarction; NSTEMI, non-ST segment elevation myocardial infarction; TC, cholesterol; TG, Triglyceride; LDL-C, low density lipoprotein-cholesterol; HDL-C, high-density lipoprotein cholesterol; BNP, B-type natriuretic peptide; BMI, body mass index; HCY, Homocysteine; HOMA-IR, homeostasis model assessment for insulin resistance DES, drug-eluting stent. 


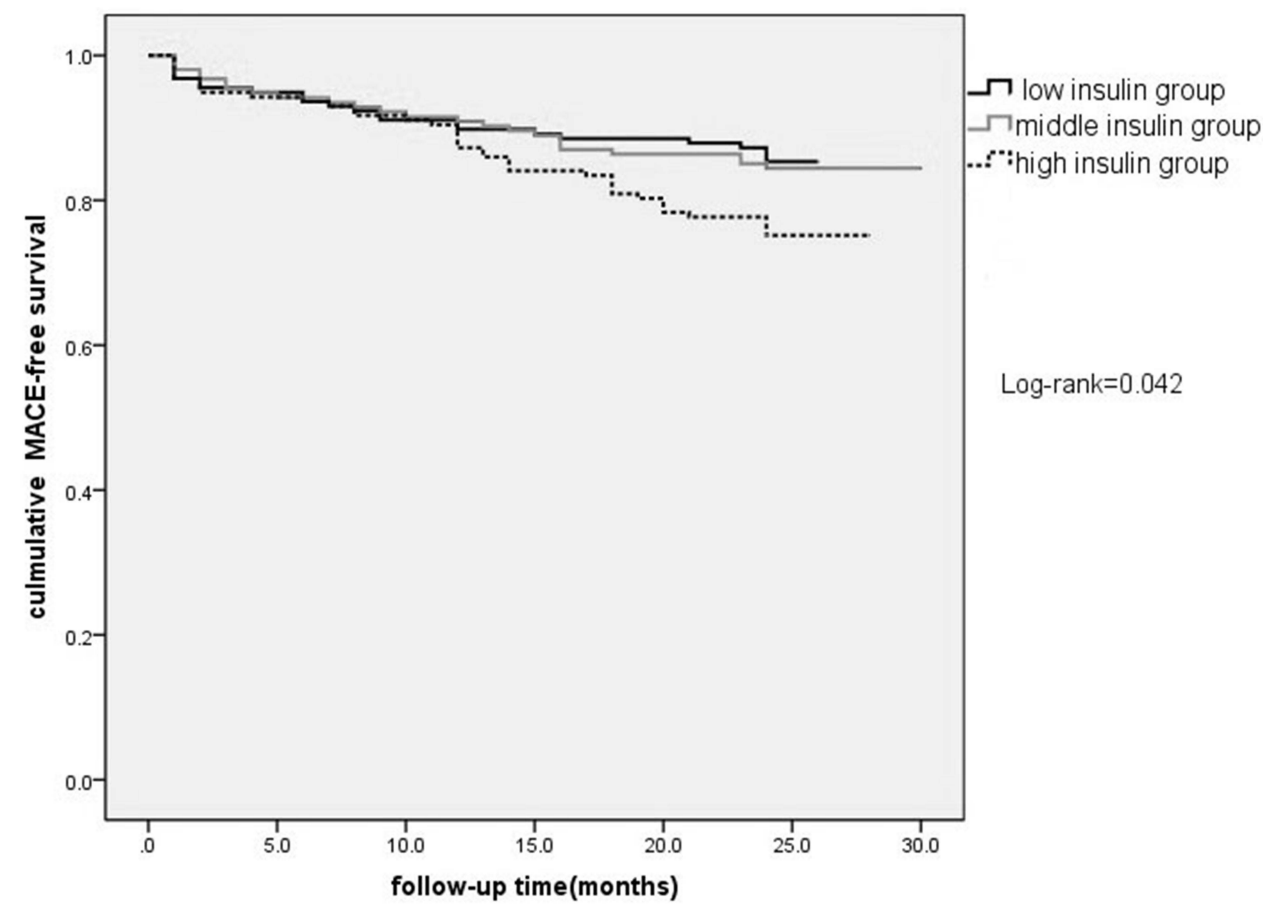

Figure I Comparison of MACE-free survival among different insulin groups.

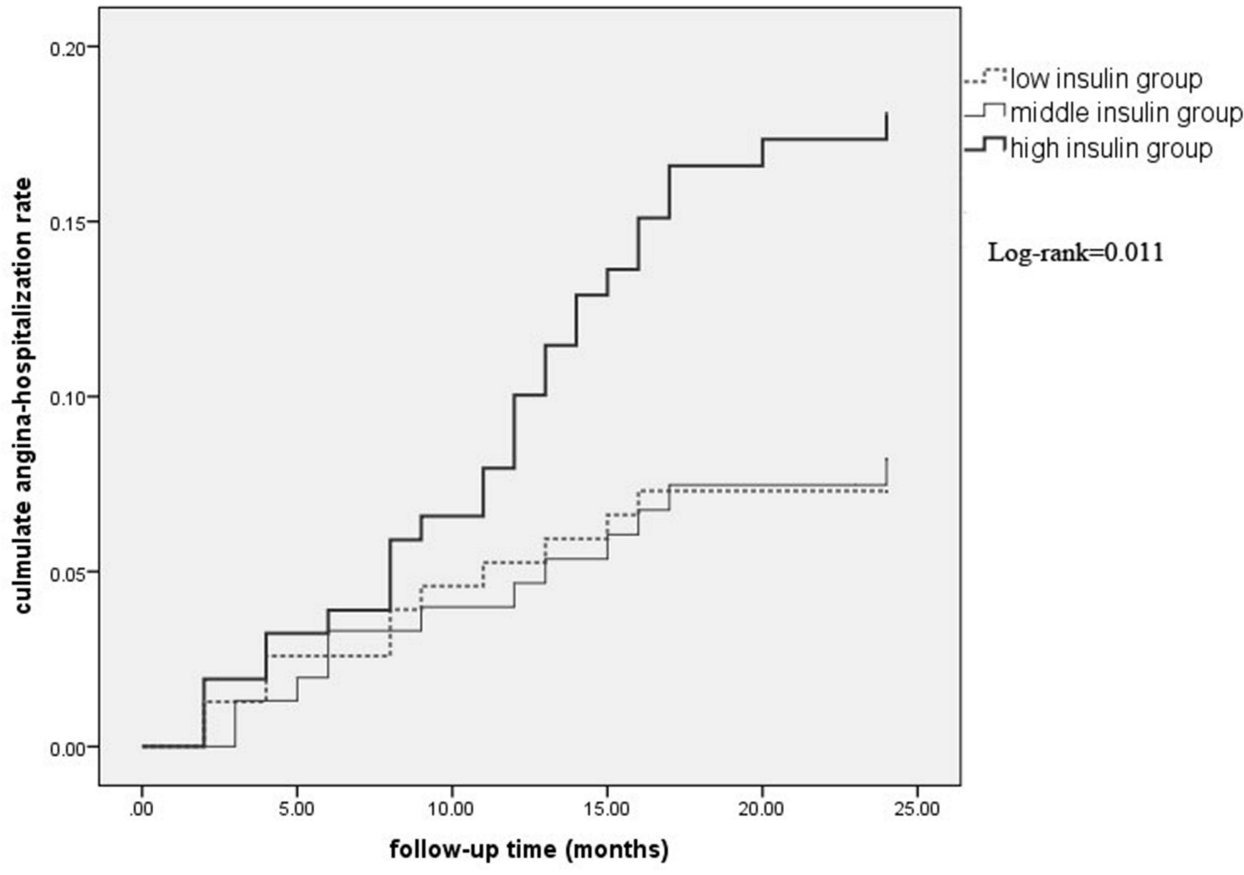

Figure 2 Comparison of angina-hospitalization among different insulin groups.

transport, promotes a compensatory increase of insulin that results in hyperinsulinemia. ${ }^{18}$ It is known that insulin has a double-phase effect on atherogenesis. Under physiological conditions, insulin stimulates the uptake of glucose and maintains glucose homeostasis. ${ }^{19}$ However, at hyper- physiological concentrations, insulin stimulates the proliferation of vascular smooth muscle cells and triggers inflammation. ${ }^{20}$ Clinical and experimental evidence suggests that hyperinsulinemia can promote obesity and endothelial dysfunction. $^{21,22}$ 
Table 3 Comparison of Clinical Outcomes After Primary PCI During 24-Month Follow-Up According to FINS Status

\begin{tabular}{|l|l|l|l|l|l|}
\hline & Low Insulin n=157 & Medium Insulin n=154 & High Insulin n= I57 & $\chi^{\mathbf{2}}$ & P \\
\hline Primary endpoint & & & & & \\
All-cause MACE & $23(22.29 \%)$ & $24(23.37 \%)$ & $39(34.39 \%)^{* \#}$ & 6.935 & 0.03 I \\
Death & $8(5.09 \%)$ & $7(4.54 \%)$ & $7(4.45 \%)$ & 1.134 & 0.567 \\
Non-fatal MI & $7(4.45 \%)$ & $9(5.84 \%)$ & $10(6.36 \%)$ & 0.319 & 0.853 \\
TVR & $8(5.09 \%)$ & $8(5.19 \%)$ & $22(14.01 \%)^{* \#}$ & 10.999 & 0.004 \\
\hline Second endpoint & & & $25(15.92 \%)^{* \#}$ & 6.177 & \\
Angina-hospitalization & $13(8.28 \%)$ & $12(7.79 \%)$ & & \\
\hline
\end{tabular}

Notes: *Compared with low-insulin group, $P<0.05$. ${ }^{\#}$ Compared with medium-insulin group, $P<0.05$.

Abbreviations: FINS, fasting insulin; MACE, major adverse cardiac events; MI, myocardial infarction; TVR, target vessel revascularization.

In the current study, we found that patients with hyperinsulinemia but without diabetes had an increased rate of MACE after undergoing PCI. As far as we know, this is the first study to discuss the direct impact of hyperinsulinemia on clinical outcomes of PCI. Our findings showed that the incidence of MACE was mainly driven by the increase in TVR. Results of multivariate analysis showed that hyperinsulinemia (as a categorical variable), but not the HOMA index, was an independent risk factor of MACE. IR is usually measured using the HOMA index, which takes into account both fasting glycemia and insulin level. Several studies have indicated that increased HOMA level is an important prognostic indicator in patients who have undergone $\mathrm{PCI}^{23,24}$ However, these studies included patients both with and without diabetes mellitus. In patients without diabetes who underwent PCI, the value of the HOMA index in predicting clinical outcomes remains controversial. Yun et al analyzed 98 consecutive patients without diabetes who underwent elective coronary angioplasty and revealed that IR (HOMA index $\geq 2.6$ ) was an independent predictor of in-hospital and 30-day MACE rates. ${ }^{25}$ Hwang et al evaluated 1-year outcomes in 229 consecutive patients with CAD and without diabetes who were treated with DES. The results showed that despite a worsening trend in angiographic outcomes in the IR group (HOMA index $\geq 2.5$ ), this did not translate into worse 1-year major clinical outcomes following PCI with DES, as compared to the non-IR group. ${ }^{26}$ Based on previous research and the current research results, hyperinsulinemia may be a more valuable predictor than the HOMA index in patients without diabetes.

Table 4 Cox Proportional Hazards Model Regression of Major Adverse Events in Patients Undergoing PCl with ACS

\begin{tabular}{|c|c|c|c|c|}
\hline & \multicolumn{2}{|l|}{ Univariate Analysis } & \multicolumn{2}{|l|}{ Multiple Analysis } \\
\hline & Coefficient $(95 \% \mathrm{CI})$ & $P$ value & Coefficient $(95 \% \mathrm{CI})$ & $P$ value \\
\hline Bifurcation & $4.428(2.68|-7.3| 3)$ & 0 & $3.394(2.033-5.667)$ & 0 \\
\hline Syntax score & $1.119(1.079-1.16)$ & 0 & $1.084(1.039-1.130)$ & 0.001 \\
\hline Stent length & $1.028(1.013-1.043)$ & 0.011 & 1.017 (1.002-1.032) & 0.03 \\
\hline Stent diameter & $0.79 \mid(0.489-\mid .28 I)$ & 0.341 & - & - \\
\hline Small vessel & $1.609(1.053-2.46 \mathrm{I})$ & 0.028 & $1.713(1.1111-2.642)$ & 0.015 \\
\hline HOMA & $1.113(0.980-1.265)$ & 0.099 & $0.980(0.825-1.093)$ & 0.473 \\
\hline Hyperinsulinemia & $2.586(1.694-3.950)$ & 0 & $1.870(1.202-2.909)$ & 0.006 \\
\hline BMI & $1.034(0.97 I-I .10 I)$ & 0.297 & - & - \\
\hline Waist line & $1.006(0.997-1.016)$ & 0.205 & - & - \\
\hline Multivessel disease & $1.765(0.978-3.185)$ & 0.059 & 1.051 (0.559-I.978) & 0.877 \\
\hline Age & 1.014 (0.99I-I.037) & 0.246 & - & - \\
\hline Hypertension & 0.897 (0.579-I.389) & 0.627 & - & - \\
\hline Smoke & $1.533(0.936-2.5 \mathrm{II})$ & 0.09 & $1.386(0.888-2.163)$ & 0.15 \\
\hline Prior MI & $0.783(0.480-1.277)$ & 0.321 & - & - \\
\hline LDL-C & $0.918(0.690-1.222)$ & 0.559 & - & - \\
\hline
\end{tabular}

Abbreviations: $\mathrm{PCl}$, percutaneous coronary intervention; ACS, acute myocardial syndrome; MI, myocardial infarction; LDL-C, low-density lipoprotein cholesterol; BMI, body mass index; HOMA, homeostasis model assessment; $\mathrm{Cl}$, confidence interval. 
Although the mechanism underlying the association of hyperinsulinemia with adverse cardiovascular outcomes has not been elucidated, it may be linked to chronic inflammation and endothelial dysfunction. Hyperinsulinemia triggers oxidative stress and inflammation in macrophages and endothelial cells. ${ }^{27}$ These cells produce pro-inflammatory cytokines and interleukins that contribute to vascular endothelial damage. ${ }^{28}$ In this study, we also found that patients with hyperinsulinemia had more frequent angina hospitalization than patients with low and medium insulin levels. The mechanism of angina may be impaired microcirculation. Impaired coronary microcirculation is frequently observed in patients with IR and type 2 diabetes mellitus. ${ }^{29}$ This impairment is driven by reduced levels of bioavailable NO. Our previous study demonstrated that hyperinsulinemia impairs endothelial progenitor cell function by down regulation of the PI-3K/Akt/eNOS pathway. ${ }^{10}$ NO is a key regulator in modulating endothelial function. Impaired NO reproduction may explain the results of the current study.

\section{Limitations}

This study has several limitations. First, this was a singlecenter study, and the sample size was relatively small. Second, this was a retrospective study and not a randomized controlled study; therefore, selection bias may be present. The third limitation is the relatively short duration (24 months) of follow-up. This may be a limiting factor in evaluating important clinical effects of hyperinsulinemia. Further investigation is necessary to clarify the impact of insulin among patients undergoing PCI.

\section{Conclusions}

In this study, we found an association between higher insulin levels and increased risk of MACE in patients with ACS who did not have diabetes. Hyperinsulinemia might be a valid predictor of clinical outcomes in patients with ACS and without diabetes who underwent PCI.

\section{Data Sharing Statement}

The datasets used during the current study are available from the corresponding author on reasonable request.

\section{Ethics Approval and Consent to Participate}

This study was approved by ethics committee of Qinhuangdao first Hospital, Hebei Medical University. And all patients provided their written informed consent.

\section{Funding}

This work was supported by S\&T program of Hebei (20377756D), Hebei Chinese medical technology project (2020377) and Hebei medical technology project (20171252).

\section{Disclosure}

The authors declare that they have no competing interests.

\section{References}

1. Gluvic Z, Zaric B, Resanovic I, et al. Link between metabolic syndrome and insulin resistance. Curr Vasc Pharmacol. 2017;15 (1):30-39. doi:10.2174/1570161114666161007164510

2. Wang HH, Lee DK, Liu M, Portincasa P, Wang DQ. Novel insights into the pathogenesis and management of the metabolic syndrome. Pediatr Gastroenterol Hepatol Nutr. 2020;23(3):189-230. doi:10.5223/pghn.2020.23.3.189

3. Grandl G, Wolfrum C. Hemostasis, endothelial stress, inflammation, and the metabolic syndrome. Semin Immunopathol. 2018;40:215-224. doi:10.1007/s00281-017-0666-5

4. Mahmoud AM, Ali M, Miranda ER, et al. Nox2 contributes to hyperinsulinemia-induced redox imbalance and impaired vascular function. Redox Biol. 2017;13:288-300. doi:10.1016/j.redox.20 17.06.001

5. Williams KJ, Wu X. Imbalanced insulin action in chronic over nutrition: clinical harm, molecular mechanisms, and a way forward. Atherosclerosis. 2016;247:225-282.

6. Erion KA, Corkey BE. Hyperinsulinemia: a cause of obesity? Curr Obes Rep. 2017;6:178-186. doi:10.1007/s13679-017-0261-z

7. Barber TM, Kyrou I, Randeva HS, Weickert MO. Mechanisms of insulin resistance at the crossroad of obesity with associated metabolic abnormalities and cognitive dysfunction. Int J Mol Sci. 2021;22 (2):546. doi:10.3390/ijms 22020546

8. da Silva AA, Do Carmo JM, Li X, Wang Z, Mouton AJ, Hall JE. Role of hyperinsulinemia and insulin resistance in hypertension: metabolic syndrome revisited. Can J Cardiol. 2020;36(5):671-682. doi:10.1016/j.cjca.2020.02.066

9. Liu R, Guan S, Gao Z, et al. Pathological hyperinsulinemia and hyperglycemia in the impaired glucose tolerance stage mediate endothelial dysfunction through miR-21, PTEN/AKT/eNOS, and MARK/ET-1 pathways. Front Endocrinol (Lausanne). 2021;23 (12):644159. doi:10.3389/fendo.2021.644159

10. Tan Q, Li Y, Li X, Zhang SY. Hyperinsulinemia impairs functions of circulating endothelial progenitor cells. Acta Diabetol. 2019;56 (7):785-795. doi:10.1007/s00592-019-01314-9

11. Baber U, Stefanini G, Giustino G, et al. Impact of diabetes mellitus in women undergoing percutaneous coronary intervention with drug-eluting stents. Circ Cardiovasc Interv. 2019;12(7):e007734. doi:10.1161/CIRCINTERVENTIONS.118.007734 
12. Wang Q, Liu H, Ding J. Outcomes of percutaneous coronary intervention in patients with coronary chronic total occlusions with versus without type 2 diabetes mellitus: a systematic review and meta-analysis. Medicine (Baltimore). 2017;96(45):e8499. doi:10.1097/MD.0000000000008499

13. Godoy LC, Lawler PR, Farkouh ME, Hersen B, Nicolau J, Rao V. Urgent revascularization strategies in patients with diabetes mellitus and acute coronary syndrome. Can J Cardiol. 2019;35(8):993-1001. doi:10.1016/j.cjca.2019.03.010

14. Patel MR, Calhoon JH, Dehmer GJ, et al. ACC/AATS/AHA/ASE/ ASNC/SCAI/SCCT/STS 2016 Appropriate Use Criteria for Coronary Revascularization in Patients With Acute Coronary Syndromes: a Report of the American College of Cardiology Appropriate Use Criteria Task Force, American Association for Thoracic Surgery, American Heart Association, American Society of Echocardiography, American Society of Nuclear Cardiology, Society for Cardiovascular Angiography and Interventions, Society of Cardiovascular Computed Tomography, and the Society of Thoracic Surgeons. J Am Coll Cardiol. 2017;69(5):570-591.

15. American Diabetes Association. Diagnosis and classification of diabetes mellitus. Diabetes Care. 2014;37(Suppl.1):S81-S90. doi:10.2337/dc14-S081

16. Li Y, Tan Q, Guo Y, et al. The influence of exercise training on endothelial function, serum irisin and inflammatory markers in the elderly with metabolic syndrome. Clin Lab. 2021;67(3):689-696. doi:10.7754/Clin.Lab.2020.200446

17. Tan Q, Wang QS, Liu DT, Zhang SY, Zhang Y, Li Y. Intravascular ultrasound-guided unprotected left main coronary artery stenting in the elderly. Saudi Med J. 2015;36(5):549-553. doi:10.15537/ smj.2015.5.11251

18. Velez M, Kohli S, Sabbah HN. Animal models of insulin resistance and heart failure. Heart Fail Rev. 2014;19:1-13. doi:10.1007/s10741013-9387-6

19. Choi JH, Gimble JM, Vunjak-Novakovic G, Kaplan DL. Effects of Hyperinsulinemia on lipolytic function of three-dimensional adipocyte endothelial co-cultures. Tissue Eng Part C Methods. 2010;16:1157-1165. doi:10.1089/ten.tec.2009.0760

20. Mackesy DZ, Goalstone ML. Insulin augments tumor necrosis factor-alpha stimulated expression of vascular cell adhesion molecule-1 in vascular endothelial cells. J Inflamm. 2011;8:34-38. doi:10.1186/1476-9255-8-34
21. Hill MA, Yang Y, Zhang L, et al. Insulin resistance, cardiovascular stiffening and cardiovascular disease. Metabolism. 2021;119:154766. doi:10.1016/j.metabol.2021.154766

22. Muniyappa R, Chen H, Montagnani M, Sherman A, Quon MJ. Endothelial dysfunction due to selective insulin resistance in vascular endothelium: insights from mechanistic modeling. Am J Physiol Endocrinol Metab. 2020;319(3):E629-E646. doi:10.1152/ ajpendo.00247.2020

23. Uetani T, Amano T, Harada K, et al. Impact of insulin resistance on post-procedural myocardial injury and clinical outcomes in patients who underwent elective coronary interventions with drug-eluting stents. JACC Cardiovasc Interv. 2012;5(11):1159-1167. doi:10.1016/j.jcin.2012.07.008

24. Kim I, Kim MC, Sim DS, et al. Effect of the metabolic syndrome on outcomes in patients aged $<50$ years versus $>50$ years with acute myocardial infarction. Am J Cardiol. 2018;122(2):192-198.

25. Yun $\mathrm{KH}$, Jeong $\mathrm{MH}, \mathrm{Kim} \mathrm{KH}$, et al. The effect of insulin resistance on prognosis of non-diabetic patients who underwent percutaneous coronary intervention. $J$ Korean Med Sci. 2006;21:212-216. doi:10.3346/jkms.2006.21.2.212

26. Hwang IK, Kim YK, Rha SW, Ra JE, Seo BS, Lee JK. Impact of insulin resistance on 1-year clinical outcomes in non-diabetic patients undergoing percutaneous coronary intervention with drug-eluting stents. J Cardiol. 2013;61:113-116. doi:10.1016/j.jjcc.2012.08.022

27. Mahmoud AM, Szczurek MR, Blackburn BK, et al. Hyperinsulinemia augments endothelin-1 protein expression and impairs vasodilation of human skeletal muscle arterioles. Physiol Rep. 2016;4(16):e12895. doi:10.14814/phy2.12895

28. Incalza MA, D’Oria R, Natalicchio A, Perrini S, Laviola L, Giorgino F. Oxidative stress and reactive oxygen species in endothelial dysfunction associated with cardiovascular and metabolic diseases. Vascul Pharmacol. 2018;100:1-19.

29. Labazi H, Trask AJ. Coronary microvascular disease as an early culprit in the pathophysiology of diabetes and metabolic syndrome. Pharmacol Res. 2017;123:114-121. doi:10.1016/j. phrs.2017.07.004

Diabetes, Metabolic Syndrome and Obesity: Targets and Therapy

Dovepress

\section{Publish your work in this journal}

Diabetes, Metabolic Syndrome and Obesity: Targets and Therapy is an international, peer-reviewed open-access journal committed to the rapid publication of the latest laboratory and clinical findings in the fields of diabetes, metabolic syndrome and obesity research. Original research, review, case reports, hypothesis formation, expert opinion and commentaries are all considered for publication. The manuscript management system is completely online and includes a very quick and fair peer-review system, which is all easy to use. Visit http://www.dovepress.com/testimonials.php to read real quotes from published authors. 